\title{
The occurrence of non-pulsating stars in the $\gamma$ Dor and $\delta$ Sct instability regions
}

\author{
Joyce A. Guzik ${ }^{1}$, Paul A. Bradley ${ }^{1}$, Jason Jackiewicz ${ }^{2}$, \\ Katrien Uytterhoeven ${ }^{3,4}$, and Karen Kinemuchi ${ }^{5}$ \\ ${ }^{1}$ Los Alamos National Laboratory \\ MS T086, Los Alamos, NM 87545 USA \\ email: joy@lanl.gov, pbradley@lanl.gov \\ ${ }^{2}$ Dept. of Astronomy, New Mexico State University \\ P.O. Box 30001, MSC 4500, Las Cruces, NM 88003 USA \\ email: jasonj $@$ @nmsu.edu \\ ${ }^{3}$ Instituto de Astrofísica de Canarias (IAC), 38200, La Laguna, Tenerife, Spain \\ ${ }^{4}$ Departamento de Astrofísica, Universidad de La Laguna, 38200 La Laguna, Tenerife, Spain \\ email: katrien@iac.es \\ ${ }^{5}$ Apache Point Observatory \\ P.O. Box 59, 2001 Apache Point Road, Sunspot, NM 88349 USA \\ email: kinemuchi@apo.nmsu.edu
}

\begin{abstract}
We examine the light curves of over 2700 stars observed in long cadence by the Kepler spacecraft as part of the Guest Observer program. Most of these stars are faint (Kepler magnitude >14), and fall near or within the effective temperature and $\log g$ range of the $\gamma$ Dor and $\delta$ Sct instability strips. We find that the pulsating stars are obvious from inspection of the light curves and power spectra, even for these faint stars. However, we find that a large number of stars are 'constant', i.e. show no frequencies in the 0.2 to $24 \mathrm{~d}^{-1}$ range above the $20 \mathrm{ppm}$ level. We discuss the statistics for the constant stars, and some possible physical reasons for lack of pulsations. On the other hand, $\gamma$ Dor and $\delta$ Sct candidates have been found in the Kepler data spread throughout and even outside of the instability regions of both types that were established from pre-Kepler ground-based observations. We revisit mechanisms to produce g- or p-mode pulsations in conditions when these modes are not expected to be unstable via the He-ionization $\kappa$ effect ( $\delta$ Sct) or convective blocking ( $\gamma$ Dor) pulsation driving mechanisms.
\end{abstract}

Keywords. stars: variables: $\delta$ Scuti, stars: variables: $\gamma$ Doradus, pulsation driving mechanisms

\section{Target selection and data reduction}

$\delta$ Sct and $\gamma$ Dor stars in the Kepler field have been monitored at an unprecedented level, and the photometric data have generated many questions for asteroseismologists to address. We present analysis of a sample of 633 stars requested for Kepler Guest Observer (GO) Cycles 2 and 3, Quarters 6-13. These stars were selected from the Kepler Input Catalog (KIC) with the criteria: $6200 \mathrm{~K}<T_{\text {eff }}<8300 \mathrm{~K} ; 3.6<\log g<4.7$; contamination factor $<10^{-2}$; Kepler Flag 0 (no prior Kepler observations).

The stars in our sample are relatively faint, with Kepler magnitudes 14-15.5. Most of the brighter stars were observed by the Kepler Asteroseismic Science Consortium (KASC) (see Uytterhoeven et al. 2011). We requested long-cadence data only (29.4 minute integration, Nyquist frequency $24.4 \mathrm{~d}^{-1}$ ). Since $\gamma$ Dor stars have frequencies of about $1 \mathrm{~d}^{-1}$, and most $\delta$ Sct stars have frequencies of $10-20 \mathrm{~d}^{-1}$, long-cadence data are adequate to identify $\gamma$ Dor and most $\delta$ Sct candidates. We used the corrected data from MAST, and a MATLAB script written by J. Jackiewicz that removes outlier points, interpolates the 
light curves to an equidistant time grid, converts Kepler flux to parts per million (ppm), and calculates the power spectrum. We flagged as 'constant' the stars with no peaks in their power spectrum above $20 \mathrm{ppm}$ for frequencies 0.2 to $24.4 \mathrm{~d}^{-1}$. We excluded stars that appear to be eclipsing binaries with stellar components that are either 'constant' or variable, once the binary signal is removed (see Gaulme \& Guzik, these proceedings). By these criteria, we find 359 'constant' stars out of the 633 stars in our sample $(\sim 60 \%)$.

\section{Distribution of 'constant' stars compared to instability regions}

We applied a $+229 \mathrm{~K}$ increase to the effective temperature given by the KIC. This offset roughly accounts for the systematic difference between the KIC $T_{\text {eff }}$ and the $T_{\text {eff }}$ found using Sloan Digital Sky Survey photometry for the temperature range of our stellar sample, as determined by Pinsonneault et al. (2012). We plotted our sample stars that are 'constant' in the $\log g-T_{\text {eff }}$ diagram, along with $\delta$ Sct and $\gamma$ Dor instability strip boundaries established from pre-Kepler ground-based observations (Rodriguez \& Breger 2001; Handler \& Shobbrook 2002) (Fig. 1). Only a small number (six) of the 'constant' stars fall within the instability regions. However, the lack of pulsations in these stars requires explanation. Some possibilities are: 1) The stars are pulsating at $\delta$ Sct frequencies above the Nyquist frequency $24.4 \mathrm{~d}^{-1}$ (no short-cadence data are available); 2 ) the stars may be pulsating in higher spherical harmonic degree $(l>3)$ modes that aren't easily detectable photometrically. However, photometric variations probably would not completely average out for modes with $l$ up to 10-20 (see Daszyńska-Daszkiewicz 2006; Balona \& Dziembowski 1999); 3) The pulsation amplitudes are at or below the noise level of these data, and might be detectable with a longer time series or by reducing noise levels, possibly using the Kepler pixel data; 4) A physical mechanism is inhibiting pulsations for some stars. For example, diffusive helium settling might turn off $\delta$ Sct pulsations. Diffusion of metals in $\gamma$ Dor stars may cause the convection zone to become too shallow to enable the convective blocking mechanism; 5) The $\log g$ or $T_{\text {eff }}$ in these 'constant' stars may be in error, so that the stars are actually outside the pulsation instability regions. If the observational errors are random, some stars may move out of the instability regions, but it is just as likely that some stars may move into the instability region.

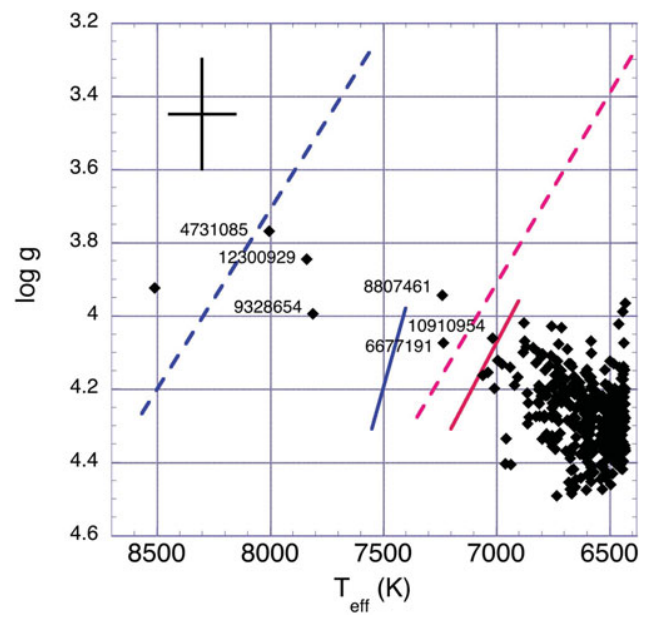

Figure 1. 'Constant' stars from Q6 - 13 data along with ground-based pulsation instability boundaries. Six 'constant' stars lie within the pulsation instability regions. 

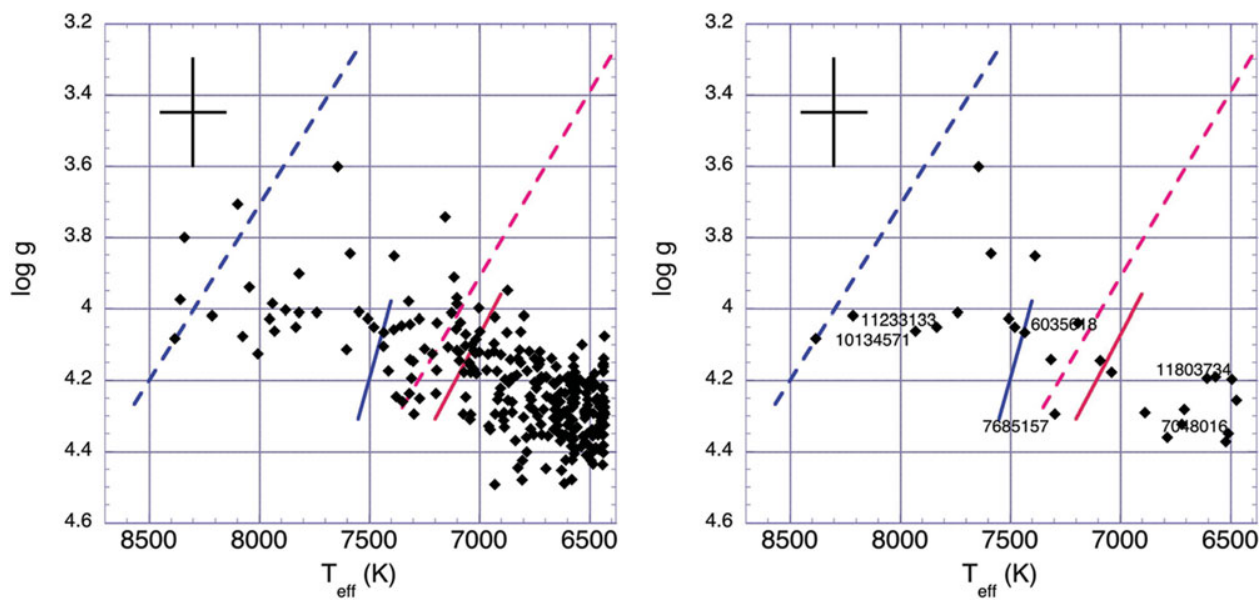

Figure 2. 'Non-constant' stars (left), and twenty-six stars with obvious $\delta$ Sct or $\gamma$ Dor pulsations (right); not all of the pulsating stars lie within their expected instability regions.

\section{Distribution of 'non-constant' stars}

Figure 2 shows the distribution of the non-constant stars, and the distribution of 26 obviously pulsating $\gamma$ Dor and $\delta$ Sct stars from this sample (see Guzik et al. 2013 for further discussion). The stars that fall outside the instability regions, or are within the instability region but pulsating in unexpected frequencies, also require explanation. Additional pulsation driving mechanisms beyond the He-ionization $\kappa$ effect ( $\delta$ Sct stars) or convective blocking ( $\gamma$ Dor stars) may be necessary to explain the oscillations of many of the Kepler stars. Some ideas that deserve further investigation include: 1) Convective driving at the top of the envelope convection zone, as in DA ZZ Ceti white dwarfs (Wu \& Goldreich 1999); 2) $\kappa$-effect from Fe concentration due to settling and levitation (Turcotte et al. 2000; Théado et al. 2009); 3) Interaction and phase offset between Fe and He ionization $\kappa$-effect (Löffler 1999); 4) Stochastic excitation (Pereira et al. 2011; Antoci et al. 2011); 5) Tidally induced pulsations (e.g., KOI-54, Welsh et al. 2011).

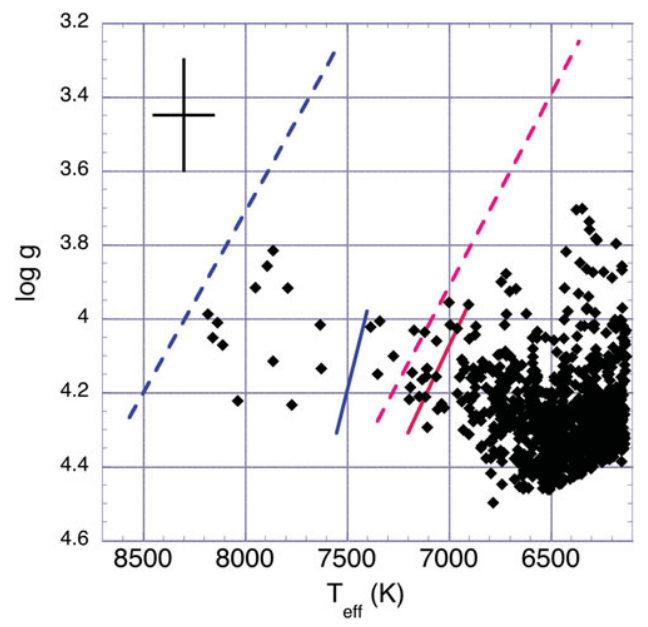

Figure 3. 'Constant' stars from Q14-16 data along with ground-based pulsation instability boundaries. 34 'constant' stars lie within the pulsation instability regions. 


\section{Q14-16 data}

We have now received data on an additional 2138 stars proposed for GO Cycle 4. This sample is not unbiased, as it was cross-correlated with stars showing variability in full-frame images. The selection criteria included stars with lower $T_{\text {eff }}$, down to $5800 \mathrm{~K}$. Using the same criteria as above, we find 984 'constant' stars (46\%); 34 out of 984 of these stars $(3.5 \%)$ are within pre-Kepler instability region boundaries (Fig. 3).

\section{Summary and future work}

In our Kepler GO Q1 - 13 data for 633 A-F main-sequence stars observed, 359 stars, or about $60 \%$, were found to be constant, defined as showing no frequencies above $20 \mathrm{ppm}$ in their light curves between 0.2 and $24.4 \mathrm{~d}^{-1}$. Only six photometrically non-varying stars are within the $\gamma$ Dor and $\delta$ Sct pulsation instability regions established from preKepler ground-based observations. Twenty-six stars, or about $10 \%$ of the non-constant stars are obvious $\gamma$ Dor or $\delta$ Sct candidates. However, many of these stars do not lie within their expected instability regions, or are outside of both instability regions. From an additional 2138 stars observed in Q14-16, we find 984 (46\%) constant stars; of these, 34 stars lie within the instability region. These data have revealed many new $\gamma$ Dor $/ \delta$ Sct candidates (see Bradley et al., these proceedings). We plan to use stellar modeling to determine whether diffusive settling can eliminate pulsations in $\gamma$ Dor or $\delta$ Sct stars, and to explore alternate pulsation driving mechanisms to explain stars that show pulsations at unexpected frequencies or lie outside of predicted instability boundaries.

\section{Acknowledgements}

The authors gratefully acknowledge support from the NASA Kepler Guest Observer program. K.U. acknowledges support from the Spanish National Plan of R\&D for 2010. This project greatly benefitted from Project FP7-PEOPLE-IRSES:ASK No. 269194.

\section{References}

Antoci, V., Handler, G., Campante, T. L., et al. 2011, Nature, 477, 570

Balona, L. A. \& Dziembowski, W. A. 1999, MNRAS, 309, 221

Daszyńska-Daszkiewicz, J., Dziembowski, W. A., \& Pamyatnykh, A. A. 2006, MemSAIt, 77, 113

Grigahcène, A., Antoci, V., Balona, L., et al. 2010, ApJ, 713, L192

Guzik, J. A., Bradley, P. A., Jackiewicz, J., Uytterhoeven, K., \& Kinemuchi, K. 2013, Astronomical Review, published on-line on 3 October 2013

Handler, G. \& Shobbrook, R. R. 2002, MNRAS, 333, 251

Löffler, W. 2000, ASP-CS, 203, 447

Pereira, T. M. D., Suárez, J. C., Lopes, I., et al. 2007, A\&A, 464, 659

Pinsonneault, M. H., An, D., Molenda-Żakowicz, J., Chaplin, W. J., Metcalfe, T. S., \& Bruntt, H. 2012, ApJS, 199, 30

Rodriguez, E. \& Breger, M. 2001, A\&A, 366, 178

Théado, S., Vauclair, S., Alecian, G., \& Le Blanc, F. 2009, ApJ, 704, 1262

Turcotte, S. 2002, ApJ, 573, L129

Uytterhoeven, K., Moya, A., Grigahcène, A., et al. 2011, A\&SA, 534, A125

Welsh, W. F., Orosz, J. A., Aerts, C., et al. 2011, ApJS, 197, 4

Wu, Y. \& Goldreich, P. 2000, ASP-CS, 203, 508 